Skeptisch eingestellt ist der Verfasser auch zum viel strapazierten sog. „Ankündigungseffekt" (Planankündigung führt zur Erhöhung privater Investitionsziele) der japanischen Planung. Dem gegenüber stellt er die These, daß, gerade wegen des starken Einflusses des Staates auf die Privatwirtschaft in Japan, die nicht plangemäße Variation der staatlichen Instrumente für die Abweichungen vom Plan sowohl im staatlichen wie im privaten Bereich verantwortlich zu machen sei. Hierin und $\mathrm{n}$ icht in der Methodik der Wirtschaftsplanung sieht er den Grund für die Diskrepanz von Plan und Wirklichkeit. „Wichtigstes Ziel der tatsächlichen Wirtschaftspolitik ist bis in die jüngste Zeit das $\mathrm{m}$ a $\mathrm{x}$ i $\mathrm{m}$ a le Wachstum des BSP (Bruttosozialprodukts) gewesen. Die Planer hatten dieses Ziel zwar allmählich ... modifiziert in Richtung auf ein o ptim a les Wachstum ..., aber die Wirtschaftspolitik hat diese Modifizierung nicht mitgemacht" (S. 115).

Die negativen Folgen des Wachstumsfetischismus sind heute nicht mehr zu übersehen. Umweltschutz und Raumordnung sind inzwischen zum innenpolitischen Gesprächsthema Nr. 1 in Japan geworden. Auf die Notwendigkeit der Entwicklung neuer Ziele und Instrumente der Wirtschaftspolitik geht der Verfasser im abschließenden 5. Kapitel ein, wobei ihm die Re-orientierung materiell von der Expansions- zur Sozial- und Strukturproblematik und methodisch vom Bruttosozialprodukt zu einem aussagefähigen Wohlstandskonzept möglich erscheint.

Der Anhang des Buches enthält u. a. eine Darstellung der Variablen und Gleichungen des Mittelfristigen Makro-Modells mit Intrapolations- und Extrapolationstests (S. 127-140) und ein umfangreiches Literaturverzeichnis über die japanische Wirtschaftsplanung (S. 160-183).

Insgesamt gesehen liefert diese Arbeit, die der Universität Tübingen als Dissertation vorgelegen hat, einen wichtigen Beitrag zum Verständnis der Struktur und Funktionsweise des dynamischen Wirtschaftssystems Japans. Daß die sophistizierten japanischen Planungsverfahren für eine Reihe hochentwickelter Volkswirtschaften von Interesse sein könnten, im Hinblick auf ihre Übertragbarkeit aber einer kritischen Prüfung unterzogen werden müßten, dafür liefert das Buch genügend Argumente.

Udo Ernst Simonis

\title{
Zaki Mustafa
}

The Common Law in the Sudan - An Account of the Justice, Equity and Good Conscience Provision

Clarendon Press, Oxford 1971, 3,50 £

Das Erscheinen dieses Buches trifft mit einem entscheidenden Einschnitt in der Rechtsentwicklung des Sudan zusammen. 1971 hat der Sudan im Rahmen einer umfassenden Kodifizierung das bis dahin geltende Zivilrecht auf der Basis des englischen „common law“ durch ein Zivilgesetzbuch abgelöst. Dieses ist im wesentlichen eine Rezeption des ägyptischen Civil Code von 1948, welcher seinerseits stark französisch-kontinental beeinflußt ist. Der Autor kündigt im Schlußkapitel (S. $239 \mathrm{ff}$.) eine solche Entwicklung bereits vorsichtig (und skeptisch) an, konnte aber leider nicht mehr zu ihr Stellung nehmen. Die Zukunft wird zeigen, ob das besprochene Buch damit Rechtsgeschichte geworden ist oder ob umgekehrt die neuen Kodifikationen (die die Abneigung eines großen Teils des sudanesischen Juristenstandes zu überwinden haben) Episode bleiben werden. Auch im (unwahrscheinlichen) Fall einer vollständigen Verdrängung der „common law“-Tradition 
wäre dem Buch jedoch kaum etwas von seinem Wert genommen. Zum einen fehlte es bisher an einer zusammenhängenden Darstellung der sudanesischen Rechtsentwicklung. Diese Darstellung bietet dieses Buch, weil der Verfasser sich bemüht, einleitend zur Rechtsentwicklung seit Beginn des anglo-ägyptischen Kondominiums, die sein eigentliches Thema ist, auch den vorhergehenden Rechtszustand auf dem Gebiet des heutigen Sudan aufzuzeigen (S. 33-38). Zum anderen ist die Rechtsentwicklung im Sudan von generellem theoretischen Interesse für die Rechtsvergleichung, insbesondere für die Erforschung der Rezeption und Adaption europäischen Rechts in Afrika.

Anders als in den britischen Kronkolonien wurde das englische Zivilrecht im Sudan nicht durch einen britischen Rechtsakt eingeführt, der das englische Recht eines bestimmten Stichtages in der Kolonie anwendbar gemacht hätte. Das wäre in einem anglo-ägyptischen „Kondominium“ trotz der faktischen britischen Alleinherrschaft auch kaum möglich gewesen. Das Zivilrecht wurde zu Beginn des Kondominiums vielmehr im wesentlichen in einer einzigen Vorschrift niedergelegt, die es den Richtern zur Pflicht macht, Recht zu sprechen nach „justice, equity, and good conscience" (S. 1 ff.). Diese Formel war, wie der Verfasser nachweist (S. 46 ff.), von ihren Vätern durchaus ernst gemeint. Sie sollte nicht lediglich die Einführung englischen Rechts verschleiern, sondern eine Anweisung an die Richter sein, aufgrund allgemeiner Rechtsprinzipien ein den lokalen Gegebenheiten angemessenes sudanesisches "common law" zu entwickeln. Mit dieser Aufgabe waren die englischen Richter, die sie erfüllen sollten, jedoch überfordert. Wie in Indien, von wo die "justice, equity and good conscience"-Formel entlehnt worden war und wo die Entwicklung ähnlich verlief (S. 5 ff.), begann sehr bald ein Heimwärtsstreben der Richter zum englischen Recht, gerechtfertigt mit der Begründung, der rechtsuchende Bürger sei am besten bedient, wenn die Richter das Recht anwenden, in dem sie ausgebildet sind (S. 94). Auf diese Weise wurde die "justice, equity and good conscience"-Formel einerseits zum Vehikel für die Rezeption englischen Rechts, andererseits aber war sie elastisch genug, den Gerichten Abweichungen vom englischen Recht zu erlauben, wenn die lokalen Verhältnisse es erforderten (S. 158 ff.).

Dieser Rechtszustand änderte sich mit der Unabhängigkeit nicht grundsätzlich. Englisches Recht blieb weiter vorherrschend (S. $183 \mathrm{ff}$.), Abweichungen unter Berufung auf sudanesische Verhältnisse wurden jedoch häufiger und systematischer (S. 212), wobei rechtssoziologisch sicher nicht ohne Einfluß war, daß inzwischen alle Richter Sudanesen waren. Sowohl einheimisches Gewohnheitsrecht (S. 217) wie islamisches Recht (S. 214/5) wurden nunmehr stärker herangezogen. Vielleicht noch wichtiger war, daß sich die Gerichte für ihre Entscheidungen immer häufiger auf sudanesische statt englische Präzedenzfälle beriefen (S. 220 ff.). Diese Entwicklung hätte zusammen mit einer inzwischen voll entwickelten stare-decisisDoktrin (Bindung des High Court an Urteile des Court of Appeal und Bindung des Court of Appeal an seine eigenen Urteile - S. 223) zu einem selbständigen sudanesischen "common law" führen können. Für ein solches sieht der Verfasser die Fundamente gelegt (S. 228), und aus dem Schlußkapitel des Buchs (S. 239 ff.) wird deutlich, daß er eine Weiterverfolgung dieser Ansätze lieber gesehen hätte als den damals geplanten und inzwischen vollzogenen Bruch mit der juristischen Vergangenheit durch die Rezeption ägyptischer Kodifikationen.

Wie vollständig dieser Bruch sein wird, ist allerdings zweifelhaft. Eine bemerkenswerte Erkenntnis, die das besprochene Buch vermittelt, ist gerade die Tatsache, 
daß das englische Recht ursprünglich nicht so sehr aufgrund des Willens des Kolonialherrn als vielmehr aufgrund von Herkunft und Ausbildung der Richter eingeführt wurde. Durch ihre Tätigkeit haben sich Technik und Terminologie des "common law" bei juristischen Berufen und Gerichten durchgesetzt (S. $167 \mathrm{ff}$., $222 \mathrm{ff}$.). Es ist sehr fraglich, ob die Einführung kontinentaler Kodifikationen diesen juristischen Stil verändern kann. Kann sie es nicht, erhebt sich die Frage, ob ein Rechtssystem mehr durch seine materiellen Rechtssätze qualifiziert wird oder durch die Art und Weise, wie die Juristen diese Sätze anwenden. Das Recht des Sudan ist damit ein faszinierendes Gebiet für die Rechtsvergleichung geworden, und man muß dankbar sein, daß für seine Erforschung mit Zaki Mustafas Buch eine so ausgezeichnete Grundlage gelegt ist. Aufgrund seiner Sachkenntnis und sorgfältigen Forschungsarbeit, insbesondere der Auswertung von Hunderten, größtenteils unveröffentlichten Entscheidungen, hat er eine Darstellung der Rechtsentwicklung im Sudan in den letzten 70 Jahren geschrieben, zu der es für andere afrikanische Länder leider kaum eine Parallele gibt.

Brun-Otto Bryde

\section{P. O'Connell}

\section{State Succession in Municipal and International Law \\ II. International Relations \\ Cambridge University Press, 1967, CXI, 430 S., £ 6 net}

Die Probleme der Staatensukzession haben zwar in den letzten Jahren etwas von der Aktualität eingebüßt, die sie während der 50er und 60er Jahre besaßen, als im Zuge der Entkolonialisierung in den ehemaligen Kolonialgebieten eine Legion neuer Staaten entstand, sie sind aber auch noch heute für den Völkerrechtler von mehr als historischem Interesse.

Der Verfasser des vorliegenden Werkes, Professor O'Connell von der Universität Adelaide, ist nicht nur ein bedeutender Völkerrechtler, sondern kann gleichzeitig als die Autorität in Fragen der Staatensukzession angesehen werden. Bereits 1956 hat er dazu eine grundlegende Monographie veröffentlicht (The Law of State Succession, Cambridge University Press 1956).

Der hier zu besprechende zweite Band seines neuen Werkes behandelt die internationalen Aspekte der Staatennachfolge. O'Connell zeigt dabei nicht nur die schon umfangreiche rechtliche Problematik der Staatensukzession auf, sondern analysiert in detaillierter Weise die noch viel größere Staatenpraxis, die in den einzelnen Ländern zu recht unterschiedlichen und oft sogar gegensätzlichen Handhabungen in vergleichbaren Fragen führte. Um einen repräsentativen Querschnitt der Vertragspraxis zu gewinnen, wurden allein über 2000 bilaterale Verträge und ihr Rechtsschicksal im Fall der Staatennachfolge untersucht.

Nach einer Darstellung der Rechtsstruktur der Staatensukzession in den verschiedenen vorstellbaren Fällen untersucht O'Connell im einzelnen die Staatenpraxis im Hinblick auf die Folgen bei Annektionen und Zessionen, bei Bildung von Staatenbund und Bundesstaat und bei der Gewährung von Unabhängigkeit an ehemals abhängige Gebiete. Gerade in diesem letzten Gebiet weist O'Connell nach, daß mit Ausnahme von Algerien, Israel und Ober-Volta alle anderen neu unabhängigen Staaten eine positive Haltung gegenüber den vorher bestehenden vertraglichen Verpflichtungen eingenommen haben. Nicht nur die bilateralen Verträge werden bezüglich der Praxis der Nachfolgestaaten untersucht, sondern ebenso die Frage 